\title{
Influence of maternal and own genotype at tanning dependence-related SNPs on sun exposure in childhood
}

\author{
Jasmine Khouja ${ }^{1,2,3^{*}}$, Sarah J. Lewis ${ }^{1,2}$ and Carolina Bonilla $a^{1,2,4}$
}

\begin{abstract}
Background: Research suggests there may be a genetic influence on the likelihood of becoming tanning dependent (TD). The way in which mothers regulate their children's sun exposure may be affected by being TD. We investigated the associations between single nucleotide polymorphisms (SNPs) related to being TD and early sun exposure.

Methods: Data from the Avon Longitudinal Study of Parents and Children (ALSPAC) were used. Associations between 17 TD related SNPs in children and their mothers and 10 sun exposure variables in children (assessed via questionnaire at age 8) were analyzed in logistic and ordinal logistic regressions. Analyses were adjusted for principal components of population structure and age (at time of questionnaire response). Models with additional adjustment for maternal or offspring genotypes were also tested. Secondary analyses included adjustment for sex and skin pigmentation.

Results: Among ALSPAC children, the rs29132 SNP in the Vesicle-associated membrane protein-associated protein A (VAPA) gene was associated with five sun exposure variables whilst the rs650662 SNP in the Opioid Receptor Mu 1 (OPRM1) gene was associated with three. The remaining SNPs did not show associations beyond what was expected by chance. After Bonferroni correction one SNP in the children was associated with an increased likelihood of using sun cream whilst in the sun at 8 years old (rs60050811 in the Spermatogenesis and Centriole Associated 1 (SPATC1) gene, OR per C allele $=1$. $34,95 \% \mathrm{Cl} 1.11-1.62, p=.003)$. In the mothers, rs650662 in OPRM1 was associated with the use of a lower factor of sun cream in their children, (OR per A allele $=0.89,95 \% \mathrm{Cl} 0.82-0.96, p=.002$ ). Whilst rs2073478 in the Aldehyde Dehydrogenase 1 Family Member B1 (ALDH1B1) gene was associated with a reduced odds of their child using a sun block or cream with a 4 star rating (OR per T allele $=0.68,95 \% \mathrm{Cl} 0.53-0.88, p=.003$ ). Similar but weaker associations were observed for the main findings in the secondary analyses.
\end{abstract}

Conclusions: We found weak evidence to suggest that genes previously associated with TD are associated with sun exposure in children of European ancestry from southwest England.

Keywords: Tanning dependenceMaternal, SNPs/single nucleotide polymorphisms, Genotypes, ALSPAC

\section{Background}

The results of a UK study suggest that tanning makes people (particularly women) feel healthier and more attractive [1]. Tanning is the action of acquiring a darker skin color by exposing the skin to ultraviolet (UV) radiation. UV exposure (either by sun exposure or tanning equipment such as sun beds) can cause skin damage which is associated with the development of all major types of skin cancer

\footnotetext{
* Correspondence: Jasmine.Khouja@bristol.ac.uk

${ }^{1}$ MRC Integrative Epidemiology Unit, University of Bristol, Bristol, UK

${ }^{2}$ Bristol Medical School: Population Health Sciences, University of Bristol,

Bristol, UK

Full list of author information is available at the end of the article
}

[2-4]. Research findings suggest that the majority of people who tan, do so despite being aware of the associated health risks [5].

Continued tanning despite knowledge of health risks could be explained by dependence. In recent research, some tanners have met criteria for substance dependence defined by the Diagnostic and Statistical Manual (DSM) and the tanning-modified Cut Down, Annoyed, Guilt Eye-opener (mCAGE) Substance Abuse Screening Tool, suggesting that individuals can become tanning dependent [6-9]. Research also suggests that tanners can experience symptoms of dependence such as withdrawal [10]. In a sample of 400

(C) The Author(s). 2018 Open Access This article is distributed under the terms of the Creative Commons Attribution 4.0 International License (http://creativecommons.org/licenses/by/4.0/), which permits unrestricted use, distribution, and 
participants from a university population in the USA, 27\% were found to be tanning dependent [11] and in a study of frequent tanners, 26\% met revised CAGE criteria and 53\% met modified DSM-IV-TR criteria for tanning dependence (TD) [12]. Additionally, TD has been found to be associated with having an increased likelihood of being sun burnt in the last year and a lower likelihood of displaying protective behaviors such as covering the skin using clothing, avoiding being exposed to the sun, and using sun block/cream [11].

The well-established health risks of exposure to UV radiation combined with the relatively high prevalence of TD among tanners presents an issue for public health which needs resolving with targeted public health messages to prevent the development of TD without blaming the individual. A recent development in the genetic underpinnings of TD may allow researchers to identify pathways to dependency; Cartmel and colleagues found an association between TD and the patched domain containing 2 (PTCHD2) gene [13]. Additionally, several single nucleotide polymorphisms (SNPs) in the Ankyrin Repeat and Kinase Domain Containing 1 (ANKK1) and Dopamine Receptor D2 (DRD2) genes which are associated with substance dependence have also been found to be associated with ever tanning [14]. Sun exposure in childhood has previously been linked to genetic scores of pigmentation/ tanning ability whereby having skin that burns instead of tanning is associated with limiting sun exposure by the use of sun block and covering the skin [15]. There may also be other genetic factors which influence childhood sun exposure besides carrying alleles which are associated with fairer skin; SNPs related to TD may influence exposure. By exploring the association between SNPs related to TD and sun exposure in children, we can determine whether there is a genetic susceptibility which may increase sun exposure in childhood. Displaying poorer protective behaviors against sun exposure in childhood could be a key indicator of the tendency to develop TD in later life as lower protective behaviors in adulthood are associated with TD [11].

However, children are not autonomous, their behavior and choices are normally limited by the actions and decisions of their parents. For this reason, exploring the association between mother's SNPs and their child's sun exposure could also be informative for public health messages to advise mothers who are at risk of being TD and whose children may be affected.

The aim of this research was to explore the association between SNPs related to TD (in both children and their mothers) and sun exposure in childhood.

\section{Methods}

\section{Study population}

The Avon Longitudinal Study of Parents and Children (ALSPAC) is a large, prospective cohort study which initially recruited 14,541 pregnant women whose expected delivery dates were from 1st April 1991 to 31st December 1992. Of the 14,062 live births, 13,988 children were alive at 1 year. This study has previously been described in detail $[16,17]$. Additionally, the study website contains details of all the data that is available through a fully searchable data dictionary: http://www. bristol.ac.uk/alspac/researchers/access/.

Questionnaire responses from 7297 mothers were collected containing measures of their child's sun exposure at age 8 . The number of participants who answered each individual question and the sociodemographic data for the sample can be found in Table 1. The analysis was restricted to participants of European ancestry to reduce the confounding effects of darker skin tones and population stratification.

\section{Measures}

The questionnaires used were created specifically for ALSPAC (Additional file 1).

Sex of the child was obtained from obstetric records. When the child was 6 years old, data was collected from the mother regarding the child's skin pigmentation. Responses were categorized as: 'always burns, never tans'; 'burns easily, rarely tans'; 'doesn't change'; 'tans easily, rarely burns'; 'always tans, never burns'; and 'can't say [their] skin is always protected'.

Measures of sun related behaviors were collected via questionnaire at 8 years old. When the child was age 8 , mothers were asked via questionnaire whether their child normally wore a hat, wore something to keep covered, used sun block or sun cream and whether they avoided the midday sun when out in the sun. Responses were categorized in the questionnaire as: 'Yes, always,' 'Yes, usually', 'Yes, sometimes', and 'No, never'. Data on the factor and star rating of the sun block or sun cream used were also gathered as well as the frequency of application. Factors of sun block or cream were categorized in the questionnaire as $1-3,4-7,8-14,15-20,21-25$, and $25+$. Categories $1-3$ and 4-7 were combined in analyses due to limited responses. Star ratings of sun block ranged from one to four with four providing the greatest protection and one providing the least. Mothers also responded to questions about whether their child had been badly sun burnt when the child was 8 years old or ever up to the age of 8 (yes or no) and the number of days they had spent more than four hours in the sun during the whole year when they were 8 years old (responses were categorized in the questionnaire as: none, $1-9,10-19,20-29,30-39,40$ or more). The age of the child in months at the time of questionnaire completion was also reported.

When the young person was 18 years old, the mother responded to a questionnaire regarding their own eye color (response options: blue, green, brown, grey, other). 
Table 1 Sex, age, sun exposure and pigmentation of the children and mothers in the study population

\begin{tabular}{|c|c|}
\hline Variable $(N)$ & $\%$ \\
\hline Female $(11,524)$ & 48.5 \\
\hline Had been badly sun burnt when aged 8 (7297) & 1.7 \\
\hline Had been badly sun burnt between ages 0 and 8 (6600) & 5.0 \\
\hline \multicolumn{2}{|l|}{$\begin{array}{l}\text { Number of days the child was in the sun for } 4 \text { or more } \\
\text { hours at } 8 \text { years }(6679)\end{array}$} \\
\hline None & 4.2 \\
\hline Less than 10 & 19.5 \\
\hline $10-19$ & 23.3 \\
\hline $10-29$ & 25.5 \\
\hline $30-39$ & 15.1 \\
\hline 40 or more & 12.4 \\
\hline \multicolumn{2}{|l|}{ Child normally wore a hat while out in the sun at 8 years (7206) } \\
\hline Never & 5.3 \\
\hline Sometimes & 36.2 \\
\hline Usually & 39.2 \\
\hline Always & 19.3 \\
\hline
\end{tabular}

Child normally wore something to keep skin covered in the sun at 8 years $(7193)$

$\begin{array}{lll}\text { Never } & 2.3 & 162 \\ \text { Sometimes } & 36.8 & 2648 \\ \text { Usually } & 44.5 & 3198 \\ \text { Always } & 16.5 & 1185\end{array}$

Child normally used sun cream whilst out in the sun at 8 years (7217)

Never

$0.9 \quad 64$

Sometimes

11.2810

Usually

$32.6 \quad 2354$

Always

55.33989

Factor of sun cream the child normally used at 8 years (7039)

$4-7$

8-14

$15-20$

$21-25$

$25+$

Star rating of sun cream child usually used at 8 years (1152)

Low

High

Child usually avoided the midday sun at 8 years (7188)

$\begin{array}{lll}\text { Never } & 8.1 & 580 \\ \text { Sometimes } & 36.1 & 2596 \\ \text { Usually } & 39.8 & 2861 \\ \text { Always } & 16.0 & 1151 \\ \text { Flwuency of sun cream application at 8 years (7059) } & & \\ \text { Once only } & 10.9 & 767 \\ \text { Every } 3 \text { to } 4 \mathrm{~h} & 43.1 & 3045\end{array}$

Table 1 Sex, age, sun exposure and pigmentation of the children and mothers in the study population (Continued)

\begin{tabular}{lll}
\hline Variable ( $)$ & $\%$ & $n$ \\
\hline Every 2 h & 34.9 & 2464 \\
Every hour & 10.1 & 711 \\
Every half hour & 1.0 & 72 \\
Child's skin pigmentation (7744) & & \\
Always burns, never tans & 0.9 & 72 \\
Burns easily, rarely tans & 9.6 & 743 \\
Doesn't change & 4.0 & 312 \\
Tans easily, rarely burns & 40.0 & 3098 \\
Always tans, never burns & 17.3 & 1337 \\
Can't say [their] skin is always protected & 28.2 & 2182 \\
Mother's eye color (3922) & & \\
Blue & 39.6 & 1553 \\
Green & 22.2 & 870 \\
Brown & 25.8 & 1011 \\
Grey & 5.8 & 227 \\
Other & 6.7 & 261 \\
Means and SDs & Mean & SD \\
Age in months at questionnaire at 8 years (7297) & 105 & 3 \\
\hline Sun exposure variables were reported by the children's mothers &
\end{tabular}

Sun exposure variables were reported by the children's mothers

\section{Genetic data}

The genotyping methods have previously been described by Bonilla and colleagues [18]. Genome-wide genotypic data for the children were generated by Sample Logistics and Genotyping Facilities at the Wellcome Trust Sanger Institute (Cambridge, UK) and the Laboratory Corporation of America (Burlington, NC, USA) with support from 23andMe (Mountain View, CA, USA) using the Illumina HumanHap550 quad chip. Using the Illumina Human660W quad array, the mothers were genotyped at CNG (Centre National de Génotypage). Quality control consisted of excluding all individuals with ambiguous sex, of non-European ancestry, extreme heterozygosity, cryptic relatedness (IBD $>0.125$ in mothers, IBD $>0.1$ in children), high missingness (missingness $>5 \%$ in mothers, missingness $>3 \%$ in children) and insufficient sample replication (IBD $<0.8)$, and all SNPs with genotyping rate $<$ $95 \%, \mathrm{MAF}<1 \%$, or out of Hardy-Weinberg equilibrium ( $p<1 \times 10^{-6}$ in mothers, $p<5 \times 10^{-7}$ in children). 477,482 SNP genotypes in common between the sample of mothers and sample of children were combined. Genotypic data was then phased with ShapeIT v2.r644, which utilizes relatedness during phasing, and was jointly imputed using IMPUTE v2.2.2 and phased haplotype data from the 1000 Genomes reference panel (phase 1, version 3, Dec 2013 release). Imputation was based on 465,740 SNPs and all 2186 reference haplotypes. 
From the genome-wide data we selected 20 SNPs for analysis (listed in Additional file 2: Table S1) based on previous findings from Cartmel and colleagues [13] and Flores et al. [14]. These SNPs did not show strong evidence for association following correction for multiple comparisons in the original studies (which had small sample sizes) but the $p$-values were below $5 \times 10^{-4}$ or were below 0.05 and were also within or around substance dependence candidate genes.

One from each pair of SNPs which were in high linkage disequilibrium $\left(\mathrm{r}^{2}>.8, n=2\right)$ were excluded from analyses. Data was unavailable for one SNP, leaving 17 SNPs out of the 20 originally selected to be included in the final analysis. The 17 SNPs were located in 13 different genes (see Table 2 and Additional file 2: Table S1). The SNPs used were well imputed $\left(r^{2}>.8\right)$, in Hardy Weinberg equilibrium and did not substantially differ in allele frequency from the allele frequency in HapMap individuals of European descent (CEU).

\section{Ethics, consent and permissions}

Ethical approval for the study was obtained from the ALSPAC Ethics and Law Committee and the Local Research Ethics Committees (http://www.bristol.ac.uk/ alspac/researchers/research-ethics/). Written informed consent was obtained from the child's mother on behalf of the mother and child.

\section{Statistical analyses}

The associations between the chosen SNPs of both the children and their mothers (exposure) and sun exposure of the children (outcome) were assessed in a series of logistic regressions. Ordinal logistic regressions were used for analysis including more than two ordinal outcome categories. Analyses were adjusted for participant age in months at the time of the questionnaire completion and the top eight principal component variables that reflect population stratification.

Additionally, models were run including both the genotype of the child and the genotype of the mother in order to adjust for the effect of both. In these fully adjusted models, principal components (1-2) of both mother and child were adjusted for.

The above models were repeated to further adjust for sex and available pigmentation variables (eye color for mothers [skin pigmentation data not available] and skin pigmentation for children). These models were considered as secondary analysis to assess whether the findings supported the primary analysis as including these covariates dramatically reduced the sample size and therefore the power to detect associations.

Results were interpreted using Bonferroni adjustment where $p<.05 / 17=.003$. The Bonferroni correction only takes account of the 17 different SNPs, the 10 sun exposures were not included in the correction as some were moderately correlated (correlation coefficients ranging from $0.01-0.57$, most coefficients> 0.20, Additional file 2:

Table 2 Number of associated sun exposure variables for children and mothers by gene before Bonferroni correction

\begin{tabular}{|c|c|c|c|c|c|c|c|c|}
\hline \multirow[b]{2}{*}{ Gene (No. SNPs tested) } & \multicolumn{8}{|c|}{ Number of sun exposure variables associated $(p<.05)$ before Bonferroni correction } \\
\hline & Child $^{a}$ & Child $^{\mathrm{b}}$ & Mother $^{a}$ & Mother ${ }^{b}$ & Child $^{c}$ & Child $^{d}$ & Mother ${ }^{c}$ & Mother $^{d}$ \\
\hline ALDH1B1 (1) & 1 & 1 & 1 & 1 & 1 & 0 & 2 & 1 \\
\hline ANKK1 (3) & 1 & 1 & 0 & 1 & 1 & 2 & 1 & 1 \\
\hline DISP3 - PTCHD2 (1) & 0 & 1 & 0 & 0 & 0 & 0 & 1 & 1 \\
\hline DRD2 (3) & 1 & 1 & 3 & 3 & 2 & 1 & 2 & 4 \\
\hline HMHB1 (1) & 0 & 1 & 0 & 1 & 1 & 0 & 2 & 1 \\
\hline KIAA1462 (1) & 0 & 0 & 0 & 1 & 3 & 1 & 0 & 2 \\
\hline LY75 (1) & 0 & 0 & 0 & 0 & 1 & 1 & 1 & 2 \\
\hline NCF4 (1) & 1 & 0 & 1 & 0 & 1 & 1 & 0 & 0 \\
\hline OPRM1 (1) & 3 & 1 & 1 & 2 & 0 & 0 & 1 & 0 \\
\hline SPATC1 (1) & 2 & 2 & 0 & 1 & 2 & 0 & 0 & 0 \\
\hline TMC7 (1) & 1 & 1 & 0 & 0 & 0 & 0 & 1 & 0 \\
\hline TMPRSS12 (1) & 0 & 1 & 1 & 1 & 0 & 1 & 0 & 0 \\
\hline VAPA (1) & 5 & 1 & 1 & 0 & 2 & 0 & 0 & 0 \\
\hline Total & 15 & 11 & 8 & 11 & 14 & 7 & 11 & 12 \\
\hline
\end{tabular}

adjusted for age and principal components

badjusted for age, principal components, and the mother's/child's genotypes

cadjusted for age, principal components, sex and pigmentation

dadjusted for age, principal components, sex, pigmentation and the mother's/child's genotypes

A total of 10 sun exposure variables were tested per SNP 
Table S2) and so were not considered independent measures. Furthermore, as these are exploratory analyses rather than hypotheses driven, the focus of these results is not on $p$-value thresholds [19].

\section{Results}

The children included in the analyses were $52 \%$ male and were aged 8 years on average (Table 1). Further demographic information reported by the mothers about the children's sun exposure as well as information relating to the pigmentation of the children and their mothers can be found in Table 1 .

Out of the 17 SNPs examined, 15 SNPs in 8 genes in the children (11 SNPs in 9 genes after adjusting for the mother's genotype) and 8 SNPs in 6 genes in the mothers (11 SNPs in 8 genes after adjusting for the child's genotype) showed evidence of association with at least one of the 10 children's sun exposure variables before applying a Bonferroni correction for multiple comparisons $(p<.05$; Table 2 and Additional file 2: Table S3). Two out of three SNPs tested in the ANKK1 gene (rs1003641 and rs7118900) and all three SNPs tested in the DRD2 gene (rs12364283, rs12422191 and rs2440390) in children or mothers were associated with at least one of the sun exposure variables (Additional file 2: Table S3). Among the ALSPAC children, five sun exposure variables were associated with one SNP (rs29132) in the VAPA gene (2 after adjusting for sex and pigmentation) and three sun exposure variables were associated with one SNP (rs650662) in the Opioid Receptor $\mathrm{Mu} 1$ (OPRM1) gene (0 after adjusting for sex and pigmentation, Table 2 and Additional file 2: Table S3). After adjusting for sex and pigmentation, one SNP in the KIAA1462 gene (rs2887510) was associated with three sun exposure variables. For information regarding the number of SNPs associated with each sun exposure variable refer to Additional file 2: Table S4.

Following adjustment for participant age and the top eight principal component variables, and Bonferroni correction for multiple comparisons $(p<.05 / 17=.003)$, only one SNP in the children, rs60050811 found in the Spermatogenesis and Centriole Associated 1 (SPATC1) gene, showed evidence of being associated with sun exposure (whether the child usually used sun cream at 8 years old ) (OR per C allele $=1.34,95 \%$ CI $1.11-1.62, p$ $=.0025)$. When adjusted for the mothers' genotype the effect size was greater, however, the evidence for this association was weakened (Table 3).

One of the mothers' SNPs (rs650662) in the OPRM1 gene was associated with a reduced odds of their child using a higher factor of sun cream at 8 years old (OR per A allele $=0.89,95 \%$ CI $0.82-0.96, p=.002$ ) and one SNP in the Aldehyde Dehydrogenase 1 Family Member B1 (ALDH1B1) gene (rs2073478) was associated with a decreased odds of their child using a sun block or cream with the highest star system rating (4) compared to any other rating $(1-3)(\mathrm{OR}$ per $\mathrm{T}$ allele $=0.68,95 \%$ CI $0.53-0$. $88, p=.003)$. However, it should be noted that there were much fewer responses to this question than others in the questionnaire and therefore only 741 participants were included in the partly adjusted model. When adjusted for the children's genotype the evidence for these associations was weakened (Table 3), but the effect remained the same.

In the secondary analyses, where sex and pigmentation were included in the analyses, the findings were similar but weakened in terms of the effect size, strength of the effect or both (Table 3). However, the inclusion of these covariates resulted in a dramatic reduction in sample size (roughly 50\%) and therefore power to detect an effect. Stratifying by sex rather than adjusting for sex indicated that the effects seen may be stronger in males but again, this further reduced the sample size and therefore the power (Additional file 2: Table S5).

To assess whether the season in which participants completed the questionnaire may have influenced responses, all the regression analyses were further adjusted for the month of questionnaire completion. There were no substantial differences between the results shown and the further adjusted results (results not shown).

\section{Discussion}

The results provide weak evidence that SNPs in the SPATC1 gene in children, and the OPRM1 and $A L D H 1 B 1$ genes in their mothers are associated with the likelihood of sun exposure in childhood.

We found more associations than would be expected by chance at a $5 \%$ alpha level (i.e., one at most) between the children's VAPA and OPRM1 SNPs and multiple sun exposure variables in the children. However, it should be noted that these behaviors are likely to be highly correlated and therefore a higher number of associations is more likely than if the behaviors were completely independent of each other. After adjustment for sex and pigmentation, there were no longer more associations than we would expect by chance. Although, this could be due to the reduction in sample size and therefore power to detect an association. SNPs in the genes ANKK1, DRD2 and PTCHD2, which were reported previously as potentially underlying TD [13, 14], did not show an association with sun exposure variables beyond what would be expected by chance, nor were they associated with the sun exposure outcomes after Bonferroni correction.

Following Bonferroni correction, a SNP (rs60050811) in the SPATC1 in the children was associated with use of sun block or cream when out in the sun at 8 years old. In the study by Cartmel et al. [13] the $C$ allele was associated with TD (OR for each additional $\mathrm{C}$ allele 
Table 3 Association of tanning dependent related single nucleotide polymorphisms and sun exposure variables

\begin{tabular}{|c|c|c|c|c|c|c|c|c|c|}
\hline \multirow[t]{2}{*}{ SNP (RA) } & \multirow[t]{2}{*}{ Outcome } & \multicolumn{4}{|c|}{ Partly adjusted } & \multicolumn{4}{|c|}{ Fully adjusted } \\
\hline & & $\mathrm{OR}$ & $95 \% \mathrm{Cl}$ & $p$ & $n$ & $\mathrm{OR}$ & $95 \% \mathrm{Cl}$ & $p$ & $n$ \\
\hline \multicolumn{10}{|l|}{ Childs SNPs } \\
\hline rs60050811 (C) & $\begin{array}{l}\text { Usually used sun block or cream when out in the sun } \\
\text { at age } 8 \text { years }\end{array}$ & 1.34 & $1.11-1.62$ & .0025 & 4983 & 1.43 & $1.09-1.88$ & .0106 & 3438 \\
\hline \multicolumn{10}{|l|}{ Mothers SNPs } \\
\hline rs650662 (A) & $\begin{array}{l}\text { Factor of sun block or cream the child usually used at } \\
\text { age } 8 \text { years }{ }^{\text {a }}\end{array}$ & 0.89 & $0.82-0.96$ & .0020 & 4524 & 0.89 & $0.81-0.99$ & .0291 & 3459 \\
\hline rs2073478 (T) & Star system rating of child's sun block or cream ${ }^{\text {b }}$ & 0.68 & $0.53-0.88$ & .0028 & 741 & 0.68 & $0.49-0.94$ & .0181 & 585 \\
\hline \multicolumn{10}{|c|}{ Analyses adjusted for sex and pigmentation } \\
\hline \multicolumn{10}{|l|}{ Childs SNPs } \\
\hline rs60050811 (C) & $\begin{array}{l}\text { Usually used sun block or cream when out in the sun } \\
\text { at age } 8 \text { years }\end{array}$ & 1.43 & $1.01-1.87$ & .0074 & 2549 & 1.29 & $0.90-1.86$ & .2651 & 1892 \\
\hline \multicolumn{10}{|l|}{ Mothers SNPs } \\
\hline rs650662 (A) & $\begin{array}{l}\text { Factor of sun block or cream the child usually used at } \\
\text { age } 8 \text { years }{ }^{\mathrm{a}}\end{array}$ & 0.86 & $0.77-0.96$ & .0057 & 2385 & 0.88 & $0.77-1.01$ & .0627 & 1919 \\
\hline rs2073478 (T) & Star system rating of child's sun block or cream ${ }^{\text {b }}$ & 0.58 & $0.40-0.82$ & .0021 & 416 & 0.58 & $0.38-0.89$ & .0131 & 338 \\
\hline
\end{tabular}

RA Risk allele (increases the risk of becoming TD, allele used based on findings from Cartmel et al. [13]); SNP Single nucleotide polymorphism

The partly adjusted model adjusted for the mothers' or child's principal components (1-8 in the mother's and child's analysis respectively), and the age of the

child (in months) when the questionnaire was completed. The fully adjusted model was adjusted for both the child and mother's SNPs and principal components

$(1-2)$ and the age of the child (in months) when the questionnaire was completed

a 0 factor $0-7 ; 1=$ factor $8-14 ; 2=$ factor $15-20 ; 3=$ factor $21-25 ; 4=25+$

${ }^{\mathrm{b}} 0=$ star rating $1-3,1=$ star rating 4

adjusted for age and sex $=4.13$ ), in ALSPAC the C allele was associated with an increase in the odds of using sun cream when in the sun. A potential explanation for this difference could be that these children may be less likely to adhere to other sun protective behaviors such as keeping covered with clothing and parents may mitigate this by ensuring their child is protected with sun cream. The rs650662 SNP in the OPRM1 gene in the mothers was associated with the factor of sun cream children normally used at 8 years. OPRM1 is associated with substance dependence [20] which supports the notion that this gene is related to TD. The gene has also been shown to be associated with skin pigmentation [21] which could explain the association as some skin types are intolerant of tanning. However, we found no clear evidence of an association between rs650662 (or rs6005081/rs2073478) and pigmentation in our study (Additional file 2: Table S6). The risk allele (A) was associated with an $11 \%$ decrease in the odds of using a higher, more protective, factor of sun cream. Interestingly, Cartmel et al. [13] found that there was an increased risk of being TD with each A allele at this SNP (OR for each additional A allele adjusted for sex and age =1.64). Similarly, the rs2073478 SNP in the ALDH1B1 gene was associated with a $32 \%$ decrease in the odds of the sun cream being used by mothers on their child having the highest and most protective star rating (4 star compared to 1-3). Again, the risk allele (T) was associated with increased risk of being TD (OR for each additional $\mathrm{T}$ allele adjusted for sex and age $=2.22$ ) in Cartmel and colleagues' findings [13]. This suggests that mothers with an increased risk of TD are using lower factors of sun cream with lower star ratings on their children which could be because they are using lower factor sun creams with lower star ratings themselves in order to enhance their tan. This is consistent with the hypothesis that mothers with TD may protect their child less from UV damage than mothers without TD. When adjusting for the mother's and children's genotypes, respectively, the evidence for all three of the above findings was weakened.

In our secondary analyses adjusting for sex and pigmentation, the results were similar but weakened. This could indicate that sex and pigmentation could be modifying the effect of the TD genotype on sun exposure which would be supported by previous evidence showing that sex and pigmentation affect indoor tanning [22]. However, the dramatic reduction in sample size that accompanied the inclusion of these covariates in the model resulted in a reduction in the power to detect an effect.

Having access to the mother's data for this study allowed us to explore the influence a mother's genotype may have on their child's sun exposure as well as the effect of the child's genotype. Interestingly the size of the effects seen in the children changed after adjustment for the maternal genotype (although the strength of evidence was weakened), whereas the effect of the mother's genotype was not modified after accounting for the child's genotype, 
suggesting that the mother's genetic background may play a role in the children's exposure to the sun. Adjusting for the mothers' and children's SNPs in these analyses provides an insight into the possible effects one may have on the other in terms of the child's behavior, but it is important to note that there is a risk of introducing collider bias when performing this adjustment [23], so the results should be interpreted with caution.

The results of this study should be carefully considered/evaluated due to the weak evidence of associations found between polymorphisms in potential TD genes and tanning related behavior in childhood. The study by Cartmel and colleagues reported a strong association of TD with the PTCHD2 gene in a gene burden test, but no SNPs showed clear evidence of association following correction for multiple comparisons [13]. Flores and colleagues on the other hand, showed associations between ever indoor tanning and substance dependence related SNPs in analyses without correction for multiple comparisons but we found no clear associations between these SNPs and the children's sun exposure [14]. This difference in findings could be due to the focus on childhood behavior in this study rather than behavior in adulthood when addictive behavior is more likely to have been established. Alternatively, the outcome of interest (ever used indoor tanning) in the study by Flores et al. [14] may have not been a good measure of tanning dependence and neither study have validated their results nor demonstrated evidence of an association after correction for multiple comparisons.

It should be noted, that this study used data from a large longitudinal data set (with a much larger sample size than the sample sizes of previous studies in the area) and therefore we had greater power to detect an effect which may explain why there are differences between our findings and those of previous reports. However, the use of maternal reports of sun-exposure may reduce the validity of the data as mothers may not be aware of their child's exposure at all times such as when they are at school. Although it could also be argued that children spend the majority of their time inside during the school day, mothers can protect their child from sun exposure during the day by applying sun cream before school, and mothers may observe sun burn when their child returns from school. At present, there is no data available on TD or indoor tanning in ALSPAC participants which prevents the direct comparison of these findings and previous findings in the area, but the longitudinal nature of the ALSPAC study provides the opportunity for future data collection to assess whether these at risk children have developed TD later in life. The cohort are now 26 years old, meaning that if we can acquire this data we could more accurately assess their TD without their mothers' mediating effects and assess the association between their genotypes and TD as well as the association between their behaviors in childhood and TD.

\section{Conclusions}

The weak findings in this study provide little support for a genetic predisposition to TD affecting sun exposure in childhood. Similarly, there was limited evidence of an association between mothers' TD genotype and their child's sun exposure. If future evidence supports these findings, it may provide reassurance to public health officials concerned about maternal influence on children's sun exposure and lead to a reframing of public health messages. Further research could explore the effect of paternal genotype on childhood behavior and whether this could mediate any of the effects found.

\section{Additional files}

Additional file 1: Questionnaire of sun exposure completed by mothers about their children at 8 years old. This file contains the questionnaire used to assess children's sun exposure at 8 years. The questionnaire was completed by the mothers about their children. (DOCX $188 \mathrm{~kb}$ )

Additional file 2: Supplementary data for the influence of maternal and own genotype at tanning dependence-related SNPS on sun exposure in childhood. This file contains Tables S1-S6 which includes a list of SNPS and relevant details) selected for analysis, a correlation matrix of sun exposure variables, a complete table of the associations between tanning dependence related SNPS and sun exposure, and the number of SNPS associated with each measured sun exposure variable prior to Bonferroni correction. Additionally, the file contains a table of associations between SNPs and pigmentation variables, and a table of associations between tanning dependence related SNPS and sun exposure stratified by sex. (XLSX $331 \mathrm{~kb})$

\section{Abbreviations}

ALSPAC: Avon longitudinal study of parents and children; ANKK1: Ankyrin repeat and kinase domain containing 1; CNG: Centre National de Génotypage; DRD2: Dopamine receptor D2; DSM: Diagnostic and statistical manual; GWAS: Genome wide association study; IBD: Identity-by-descent; MAF: Minor allele frequency; mCAGE: The tanning-modified cut down, annoyed, guilt eye-opener substance Abuse Screening tool; OPRM1: Opioid receptor Mu 1; PTCHD2: Patched domain containing 2; SNP: Single nucleotide polymorphism; SPATC1: Spermatogenesis and centriole associated 1; TD: Tanning dependence; UV: Ultraviolet

\section{Acknowledgements \\ We are extremely grateful to all the families who took part in this study, the midwives for their help in recruiting them, and the whole ALSPAC team, which includes interviewers, computer and laboratory technicians, clerical workers, research scientists, volunteers, managers, receptionists and nurses. The UK Medical Research Council and Wellcome Trust (Grant ref.: 102215/2/ 13/2) and the University of Bristol provide core support for ALSPAC. This publication is the work of the authors and they will serve as guarantors for the contents of this paper.}

\section{Funding}

The authors are supported by the UK Medical Research Council Integrative Epidemiology Unit at the University of Bristol (MM_UU_12013/6). The UK Medical Research Council and the Wellcome Trust [Grant ref:: 102215/2/13/2] and the University of Bristol provide core support for ALSPAC. JK is a PhD student funded by the MRC Integrative Epidemiology Unit at Bristol University. 


\section{Availability of data and materials}

The datasets generated and/or analyzed during the current study are available from the corresponding author on reasonable request, subject to the ALSPAC study executive data access procedures, as specified on the ALSPAC website (http://www.bristol.ac.uk/alspac/researchers/access/) for researchers who meet the criteria for access to confidential data.

\section{Authors' contributions}

$\mathrm{CB}$ conceived, and $\mathrm{CB}$ and $\mathrm{SL}$ designed the study. JK and CB were responsible for data analysis. All authors (JK, CB and $\mathrm{SL}$ ) contributed to the interpretation of the results and the production of the manuscript. All authors (JK, CB and SL) read and approved the final manuscript.

\section{Ethics approval and consent to participate}

Ethical approval for the study was obtained from the ALSPAC Ethics and Law Committee and the Local Research Ethics Committees (http://www.bristol.ac.uk/ alspac/researchers/research-ethics/. Written informed consent was obtained from the child's mother on behalf of the mother and child.

\section{Competing interests}

The authors declare that they have no competing interests.

\section{Publisher's Note}

Springer Nature remains neutral with regard to jurisdictional claims in published maps and institutional affiliations.

\section{Author details}

${ }^{1}$ MRC Integrative Epidemiology Unit, University of Bristol, Bristol, UK. ${ }^{2}$ Bristol Medical School: Population Health Sciences, University of Bristol, Bristol, UK. ${ }^{3}$ School of Experimental Psychology, University of Bristol, Bristol, UK. ${ }^{4}$ Departamento de Medicina Preventiva, Faculdade de Medicina, Universidade de São Paulo, São Paulo, Brazil.

Received: 15 November 2017 Accepted: 4 April 2018

Published online: 12 April 2018

\section{References}

1. Boyle R, O'Hagan AH, Donnelly D, Donnelly C, Gordon S, McElwee G, Gavin A. Trends in reported sun bed use, sunburn, and sun care knowledge and attitudes in a U.K. region: results of a survey of the Northern Ireland population. Br J Dermatol. 2010;163:1269-75.

2. Mogensen $M$, Jemec $G B$. The potential carcinogenic risk of tanning beds: clinical guidelines and patient safety advice. Cancer Manag Res. 2010;2:277-82.

3. Fatiha El G, Robert B, Kurt S, Yann G, Béatrice $S$, Véronique B, Lamia B-T, Neela G, Crystal F, Laurent G, et al. A review of human carcinogens-part D: radiation. The Lancet Oncol. 2009;10:751-2.

4. Le Clair MZ, Cockburn MG. Tanning bed use and melanoma: establishing risk and improving prevention interventions. Prev Med Rep. 2016;3:139-44.

5. Knight JM, Kirincich AN, Farmer ER, Hood AF. Awareness of the risks of tanning lamps does not influence behavior among college students. Arch Dermatol. 2002;138:1311-5.

6. Nolan BV, Taylor SL, Liguori A, Feldman SR. Tanning as an addictive behavior: a literature review. Photodermatol Photoimmunol Photomed. 2009;25:12-9.

7. Harrington CR, Beswick TC, Leitenberger J, Minhajuddin A, Jacobe HT, Adinoff B. Addictive-like behaviours to ultraviolet light among frequent indoor tanners. Clin Exp Dermatol. 2011;36:33-8.

8. Heckman CJ, Darlow S, Kloss JD, Cohen-Filipic J, Manne SL, Munshi T, Yaroch AL, Perlis C. Measurement of tanning dependence. J Eur Acad Dermatol Venerol. 2014;28:1179-85.

9. Farhat EK. Top-accessed article: UV light tanning as a type of substancerelated disorder. Arch Dermatol. 2012;148:1182

10. Kaur M, Liguori A, Lang W, Rapp SR, Fleischer AB Jr, Feldman SR. Induction of withdrawal-like symptoms in a small randomized, controlled trial of opioid blockade in frequent tanners. J Am Acad Dermatol. 2006;54:709-11.

11. Heckman CJ, Egleston BL, Wilson DB, Ingersoll KS. A preliminary investigation of the predictors of tanning dependence. Am J Health Behav. 2008;32:451-64.

12. Warthan MM, Uchida T, Wagner RF Jr. UV light tanning as a type of substance-related disorder. Arch Dermatol. 2005;141:963-6.
13. Cartmel B, Dewan A, Ferrucci LM, Gelernter J, Stapleton J, Leffell DJ, Mayne ST, Bale AE. Novel gene identified in an exome-wide association study of tanning dependence. Exp Dermatol. 2014;23:757-9.

14. Flores KG, Erdei E, Luo L, White KA, Leng S, Berwick M, Lazovich D. A pilot study of genetic variants in dopamine regulators with indoor tanning and melanoma. Exp Dermatol. 2013;22:576-81.

15. Bonilla C, Ness AR, Wills AK, et al. Skin pigmentation, sun exposure and vitamin D levels in children of the Avon Longitudinal Study of Parents and Children. BMC Public Health. 2014;14:597.

16. Boyd A, Golding J, Macleod J, Lawlor DA, Fraser A, Henderson J, Molloy L, Ness A, Ring S, Davey Smith G. Cohort profile: the 'children of the 90s'-the index offspring of the Avon longitudinal study of parents and children. Int J Epidemiol. 2013;42:111-27.

17. Fraser A, Macdonald-Wallis C, Tilling K, Boyd A, Golding J, Davey Smith G, Henderson J, Macleod J, Molloy L, Ness A, et al. Cohort profile: the Avon longitudinal study of parents and children: ALSPAC mothers cohort. Int J Epidemiol. 2013:42:97-110.

18. Bonilla C, Lewis SJ, Rowlands MA, Gaunt TR, Davey Smith G, Gunnell D, Palmer T, Donovan JL, Hamdy FC, Neal DE, et al. Assessing the role of insulin-like growth factors and binding proteins in prostate cancer using Mendelian randomization: genetic variants as instruments for circulating levels. Int J Cancer. 2016:139:1520-33.

19. Sterne JAC, Davey Smith G. Sifting the evidence - what's wrong with significance tests? Brit Med J. 2001;322:226.

20. Zhang H, Luo X, Kranzler HR, Lappalainen J, Yang BZ, Krupitsky E, Zvartau E, Gelernter J. Association between two $\mu$-opioid receptor gene (OPRM1) haplotype blocks and drug or alcohol dependence. Hum Mol Genet. 2006; 15:807-19.

21. Quillen EE, Bauchet M, Bigham AW, Delgado-Burbano ME, Faust FX, Klimentidis YC, Mao X, Stoneking M, Shriver MD. OPRM1 and EGFR contribute to skin pigmentation differences between indigenous Americans and Europeans. Hum Genet. 2011;131:1073-80.

22. Li WQ, Cho E, Han J, Wu S, Qureshi AA. Pigmentary traits and use of indoor tanning beds in a cohort of women. Brit J Dermatol. 2017;176:526-30.

23. Richmond RC, Timpson NJ, Felix JF, Palmer T, Gaillard R, McMahon G, Davey Smith G, Jaddoe W, Lawlor DA: Using genetic variation to explore the causal effect of maternal pregnancy adiposity on future offspring adiposity: a Mendelian randomisation study. PLoS Med. 2017;14(1):e1002221.

\section{Ready to submit your research? Choose BMC and benefit from:}

- fast, convenient online submission

- thorough peer review by experienced researchers in your field

- rapid publication on acceptance

- support for research data, including large and complex data types

- gold Open Access which fosters wider collaboration and increased citations

- maximum visibility for your research: over $100 \mathrm{M}$ website views per year

At BMC, research is always in progress.

Learn more biomedcentral.com/submissions 